\title{
AN IN SILICO STUDY OF NARINGENIN-MEDIATED NEUROPROTECTION IN PARKINSON'S DISEASE
}

\author{
SAURABH KUMAR JHA, PRAVIR KUMAR* \\ Department of Biotechnology, Molecular Neuroscience and Functional Genomics Laboratory, Delhi Technological University (Formerly \\ DCE), Delhi - 110 042, India. Email: pravirkumar@dce.edu
}

Received: 25 March 2017, Revised and Accepted: 28 April 2017

\begin{abstract}
Objective: Naringenin is a dietary biomolecule with broad spectrum of activities which protects neurons from various neurotoxic insults and improves cognition and motor function in neurodegenerative diseases. DJ-1 has both, ubiquitin E3 ligase as well as chaperonic activity, and loss of ubiquitin E3 ligase activity of DJ-1 has been found to be associated with familial Parkinson's disease (PD). Naringenin induced E3 ligase activity of DJ-1 which can have possible clinical relevance in PD.
\end{abstract}

Methods: Various in silico parameters such as phylogenetic analysis, homology modeling, active site prediction, and molecular docking studies using AutoDock 4.2.1 and LIGPLOT1.4.5 were carried out.

Results: Three-dimensional structure of DJ-1 was generated and Ramachandran plot was obtained for quality assessment. RAMPAGE displayed $99.5 \%$ of residues in the most favored regions. $0 \%$ residues in additionally allowed and $0.5 \%$ disallowed regions of DJ-1 protein. Further, initial screenings of the molecules were done based on Lipinski's rule of five. CastP server used to predict the ligand binding site suggests that this protein can be utilized as a potential drug target. Finally, we have found naringenin to be most effective among four biomolecules in modulating DJ-1 based on minimum inhibition constant, Ki, and highest negative free energy of binding with maximum interacting surface area in the course of docking studies.

Conclusion: Our study suggests that based on different in silico parameters and molecular docking studies, naringenin can provide a new avenue for PD therapeutics.

Keywords: Parkinson's disease, Ubiquitin E3 ligase, DJ-1, Molecular docking, Naringenin.

(C) 2017 The Authors. Published by Innovare Academic Sciences Pvt Ltd. This is an open access article under the CC BY license (http://creativecommons. org/licenses/by/4. 0/) DOI: http://dx.doi.org/10.22159/ajpcr.2017.v10i8.18709

\section{INTRODUCTION}

Parkinson's disease (PD) is the second most common neurodegenerative disorder characterized by dopamine depletion in the striatum. PD is characterized by bradykinesia, tremor, rigidity, and weakening of postural reflexes [1]. The dramatic loss of neuromelanin containing dopaminergic neurons is convoyed by the presence of Lewy bodies in the remaining neurons. The hallmark of PD includes fibrillar cytoplasmic inclusions comprising aggregated and abnormally accumulated proteins, the most prominent being $\alpha$-synuclein, neurofilaments, ubiquitin, and ubiquitinated proteins [2]. In addition, at least 13 loci and nine genes have been proposed to be linked with PD, but only six genes are widely accepted to be associated with Mendelian forms of the disease [3]. Mutations in these genes potentially lead to autosomal dominant ( $\alpha$-synuclein and LRRK2) or autosomal recessive PD (Parkin, PINK1, DJ1, and ATP13A2). Recent epidemiological studies have shown that $<10 \%$ of PD cases are of familial origin with the majority being sporadic [4]. The sporadic form of PD is caused by mutated DJ1 which shows reduced nuclear localization and translocation to mitochondria [5]. DJ-1 is ubiquitously expressed in a number of pathways associated with PD pathogenesis and has ubiquitin E3 ligase activity which also reduces $\alpha$-synuclein aggregation [6]. Although mutations associated with DJ-1 lead to onset of familial PD, the exact mechanism behind the pathogenesis is still unknown [7]. Furthermore, various studies have advocated that several compounds of plant origin possess neuroprotective properties, however, their mode of action has not been clearly defined [8]. In this study, we have initially screened four biomolecules, namely, naringenin, quercetin, resveratrol, and sesamol based on Lipinski's rule of five. These biomolecules are found in fruits and vegetables and have various beneficial effects such as antioxidative, activation of survival genes and signaling pathways, chelation of transition metal, regulating mitochondrial function, and modulating neuroinflammation. Further, these biomolecules interact with significant neuronal signaling cascades that lead to inhibition of apoptosis enhanced by the neurotoxic species and promote neuronal endurance and differentiation [9]. They selectively target a number of protein kinase and lipid kinase signaling cascades, importantly, the PI3K/Akt and MAP kinase pathways which modulate prosurvival transcription factors and gene expression [10]. Interestingly, naringenin treatment prominently suppressed oxidative stressors, improved levels of enzymatic antioxidants, and neurotransmitter significantly [11]. In this study, biomolecules which exhibit neuroprotective activities were subjected to docking simulations using AutoDock 4.2.1. The preliminary investigation revealed naringenin as the best potential biomolecule among all given four biomolecules based on minimum inhibition constant, Ki, and highest negative free energy of binding with maximum interacting surface area with the active site of DJ-1 in a course of docking study. Based on in silico experimentation, naringenin is a seemingly new prospect for therapeutic intervention in PD. Therefore, a comprehensive understanding of the molecular mechanism associated with naringenin-mediated therapeutics could contribute toward clinical significance in PD biology.

\section{METHODS}

Retrieval of ubiquitin E3 ligase DJ-1 and their function recognition The amino acid sequence of ubiquitin E3 ligase DJ-1 with accession numbers 4ZGG_A was retrieved from NCBI database and used for homology search using basic local alignment search tool (BLAST). Protein functional elucidation was done using Interproscan server (http://www.ebi.ac.uk/interpro/search/sequence-search/) [12]. 
Phylogenetic relationship and physicochemical properties For multiple sequence analysis, Muscle software (http://www.ebi. ac.uk/Tools/msa/muscle/) was used and phylogenetic tree was constructed using muscle software based on neighbor joining plot without distance correction. ProtParam (http://web.expasy.org/ protparam/) was used to predict physicochemical properties [13]. The parameters computed by ProtParam included the molecular weight, theoretical PI, aliphatic index, and grand average of hydropathicity (GRAVY).

Homology modeling, visualization, and quality assessment of three-dimensional (3D) structure of ubiquitin E3 ligase DJ-1 Homology modeling was used to determine the 3D structure of DJ-1 isoforms. Templates with PDB ID 4ZGG were retrieved for DJ-1 proteins from PDB. The protein structure prediction server Swissmodel (http://swissmodel.expasy.org/) was used for homology model construction [14]. Once the 3D structure of proteins was generated, structural evaluation and stereochemical analysis were performed using RAMPAGE (http://www.mordred.bioc.cam. ac.uk/ rapper/rampage.php). Errat server (http://nihserver.mbi. ucla.edu/ERRATv2/) was used to find the accuracy of the structure and visualization of determined structures was performed using PyMOL viewer.

\section{Ligand optimization}

Reported ligand molecules (naringenin, quercetin, resveratrol, and sesamol) along with their physical and chemical properties were retrieved from PubChem compound database (http://www.pubchem. ncbi.nlm.nih.gov/). SDF files of ligands were converted in PDB file with the help of Open Babel tool that could be used for docking study. Visualization of the molecular structure of compounds was done using PyMOL viewer.

\section{Lipinski's filter analysis of screened drugs}

An online tool Lipinski's filter (http://www.scfbio-iitd.res.in/software/ drugdesign/lipinski.jsp) was used to retrieve the information about druglikeness of drugs with the help of Lipinski's rule of five. Lipinski's rule (or Lipinski's rule of five) helps to differentiate drug and non-druglike molecules [15]. It is used to identify the possibility of success or failure due to druglikeness for molecules fulfilling with two or more of the following rules: (a) Molecular mass should be $<500 \mathrm{Da}$, (b) high lipophilicity (expressed as $\log \mathrm{P}<5$ ), (c) $<5$ hydrogen bond donors, (d) $<10$ hydrogen bond acceptors, and (e) molar refractivity should be between 40 and 130 .

\section{Active site prediction}

CastP server (http://www.sts.bioe.uic.edu/castp/) was used to predict the active sites of protein [16]. CastP could also be used to measure area, circumference of mouth openings of each binding site insolvent, and molecular accessible surface. PDB file of protein was upload in the server and it showed the ligand binding sites present in protein and the site with maximum surface area and maximum surface volume was selected and all the amino acid residues involved in binding with ligands were retrieved.

\section{Preparation of protein and ligand molecules}

Preparation of protein involves the addition of polar hydrogen atoms, addition of charge, and removal of any miscellaneous structures from the protein molecule by Autodock 4.2.1, whereas ligand preparation involves the addition of charge.
Molecular docking analysis

Prepared and optimized structures of ligands and proteins were ultimately used for molecular docking using Autodock 4.2.1 for predicting the possible protein-ligand interactions and the results that include the understanding of the association that involves H-bonding and hydrophobic interactions were analyzed using LIGPLOT1.4.5, a program to generate schematic diagrams of protein-ligand interactions.

\section{RESULTS}

Retrieval of ubiquitin E3 ligase DJ-1 and their functional elucidation

Based on functional domain sequence of well-characterized gene/protein, homology search was performed using BLAST. We have successfully hunted 5 isoforms of protein DJ-1 (Table 1) on the basis of families and domains identified from Interproscan results. Interproscan study revealed that all homologues proteins for DJ-1, all homologous proteins were belonging to DJ-1 family (IPR006287), glutamine amidotransferase-like domain (IPR029062), and DJ-1/PfpI domain (IPR002818), respectively (Fig. 1).

\section{Phylogenetic relationship and physicochemical properties}

For multiple sequence analysis, Muscle software was used and found that amino acid residues were conserved in most of the isoforms of the ubiquitin E3 ligase DJ-1. Phylogenetic study of DJ-1 hunted proteins revealed that PD (autosomal recessive, early-onset) 7 and protein DJ-1 were differing from others (Fig. 2a and b). However, another Chain A (crystal structure of aggregated form of DJ-1 Chain A) and Chain A (crystal structure of E18a human DJ-1) were in same cluster as share more homology while crystal structure of human DJ-1 was in another cluster. ProtParam showed that molecular weight of DJ-1 was 19848.7 $\mathrm{Da}$ and an isoelectric point of DJ-1 was 6.37 which indicate that DJ-1 was negatively charged, respectively. Furthermore, GRAVY index of -0.47 for DJ-1 is indicative of hydrophilic (Table 2).

Homology modeling, 3D structure visualization, and quality assessment of retrieved proteins

Prediction of 3D structure of proteins provides us precise functional information of how proteins interact and localize in their stable conformation. Homology modeling is one of the most common

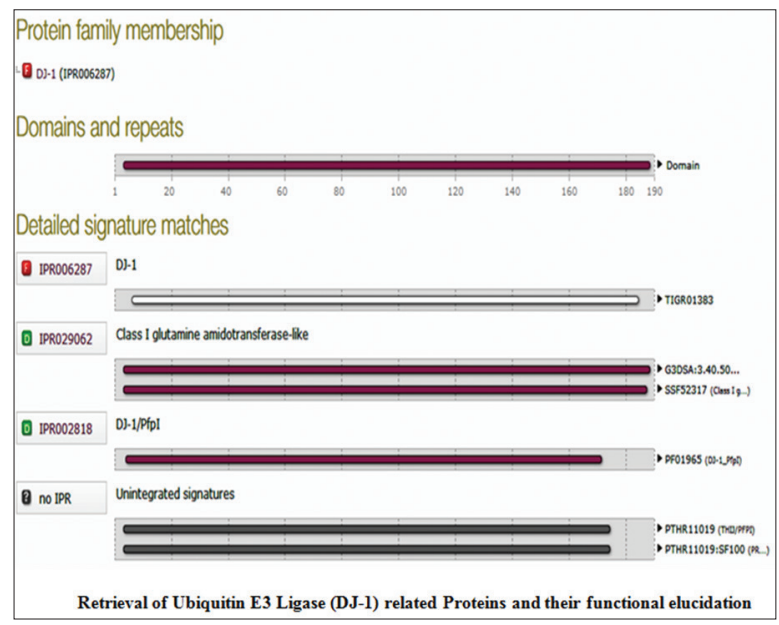

Fig. 1: Interproscan result for DJ-1

Table 1: Hunted DJ-1-related proteins

\begin{tabular}{lllll}
\hline S.No. & Accession & Protein & Score & Identity (\%) \\
\hline 1 & NP_009193.2 & Protein DJ-1 & 368 & 98 \\
2 & 3BWE_A & Chain A, crystal structure of aggregated form of DJ-1 & 365 & 100 \\
3 & 40Q4_A & Chain A, crystal structure of E18a human DJ-1 & 361 & 97 \\
4 & 1J42_A & Chain A, crystal structure of human DJ-1 & 363 & $9.00 \mathrm{e}-127$ \\
5 & ADQ32403.1 & Parkinson disease (autosomal recessive, early-onset) 7 & 364 & 97 \\
\hline
\end{tabular}


structure prediction tools in structural genomics and proteomics. The best matching template was selected for the target protein on the basis of sequence homology using PDB advance blast. Template is experimentally determined $3 \mathrm{D}$ structure of protein that shares sequence similarity with target sequence. Template showed sequence identity of $100 \%$ for DJ-1 isoforms. 3D structure of DJ-1 was generated using Swiss-model server. The Z-score is indicative of overall model quality and is used to check whether the input structure is within the range of scores typically found for native proteins of similar size. Z-score of the template and query model was obtained by Swissmodel. Z-score for DJ-1 has been shown in (Table 3), suggesting a good structure.

3D structure of DJ-1 was generated. Even though there were no steric clashes in the structure generated, these were assessed for geometric and energy aspects (Fig. 3a). Ramachandran plot was used to check the reliability of predicted 3D structure of ubiquitin E3 ligase proteins DJ-1. RAMPAGE checks the stereochemical quality of a protein structure by analyzing residue-by-residue geometry and overall structure geometry. Ramachandran plots were obtained for DJ-1 for quality assessment (Fig. 3b). RAMPAGE displayed 99.5\% of residues in the most favored regions, $0 \%$ residues in additionally allowed, and $0.5 \%$ disallowed regions of DJ-1 protein. Errat server was used to find the accuracy of the model. Result of Errat showed $98.844 \%$ of accurate structure for DJ-1 proteins.

Physicochemical properties and Lipinski's filter analysis retrieved of ligands

Initial screening of the molecules was done on the basis of Lipinski's rule of five (Fig. 4). Lipinski's filter analysis revealed that all these molecules (naringenin, quercetin, resveratrol, and sesamol) could act as a drug as they meet the criteria of Lipinski's rule of five. Further, the physicochemical properties of these selected biomolecules have been outlined in (Table 4).

Active site prediction and molecular docking analysis of DJ-1 with identified molecules

CastP server was used to predict the ligand binding site. This server calculates the possible active sites from the 3D atomic coordinates of the proteins. For DJ-1, residues involved in ligand binding site, site volume, and volume of protein for 33 active sites were predicted (Fig. 3c). Among the 33 binding sites obtained from CastP for DJ-1, site 33 was highly conserved within the active site of the protein. The predicted site 33 comprised $435.6 \AA^{3}$ site volume out of the $1723.5 \AA^{3}$ of protein volume. The residues in site 33 are shown in (Table 6).

Molecular docking pattern of DJ-1 with screened molecules (naringenin, quercetin, resveratrol, and sesamol) has been identified and depicted in (Fig. 5). On the basis of docking analysis, interacting compounds with minimum binding constant and highest negative free energy of binding

Table 2: Physicochemical properties of DJ-1

\begin{tabular}{ll}
\hline Properties & DJ-1 \\
\hline Molecular formula & $\mathrm{C}_{874} \mathrm{H}_{1448} \mathrm{~N}_{242} \mathrm{O}_{268} \mathrm{~S}_{8}$ \\
Molecular weight (Da) & 19848.7 \\
Theoretical PI & 6.37 \\
Aliphatic index & 99.11 \\
GRAVY & -0.047 \\
\hline
\end{tabular}

GRAVY: Grand average of hydropathicity

Table 3: Swiss-model server result showing template structure used in homology modeling, sequence identity, and quality score of the model generated

\begin{tabular}{lllll}
\hline $\begin{array}{l}\text { Gene } \\
\text { name }\end{array}$ & $\begin{array}{l}\text { Modeled } \\
\text { residue range }\end{array}$ & $\begin{array}{l}\text { Based on } \\
\text { template }\end{array}$ & $\begin{array}{l}\text { Sequence } \\
\text { identity (\%) }\end{array}$ & $\begin{array}{l}\text { QMEAN } \\
\text { Z-score }\end{array}$ \\
\hline DJ-1 & $2-185$ & 4ZGG & 100 & -0.42 \\
\hline
\end{tabular}

are most effective. Docking calculation of DJ-1 has been presented in (Table 5).

Binding site residues of DJ-1 interacting with naringenin, quercetin, resveratrol, and sesamol were found to be the same as the residues involved in their respective catalytic sites. Interacting residues of DJ-1 with naringenin, quercetin, resveratrol, and sesamol along with

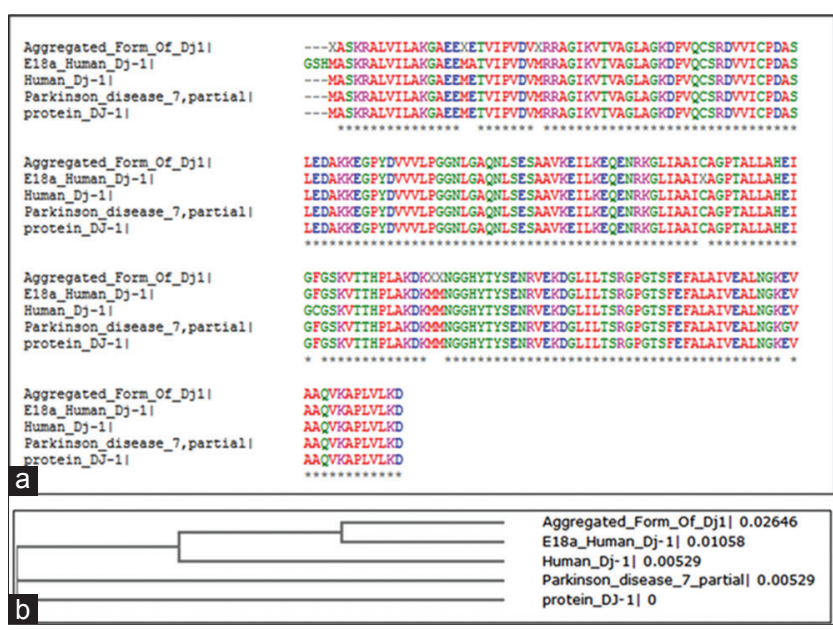

Fig. 2: (a) Multiple sequence alignment of all DJ-1 isoforms and (b) Tree generation for DJ-1 using neighbor joining plot without distance correction

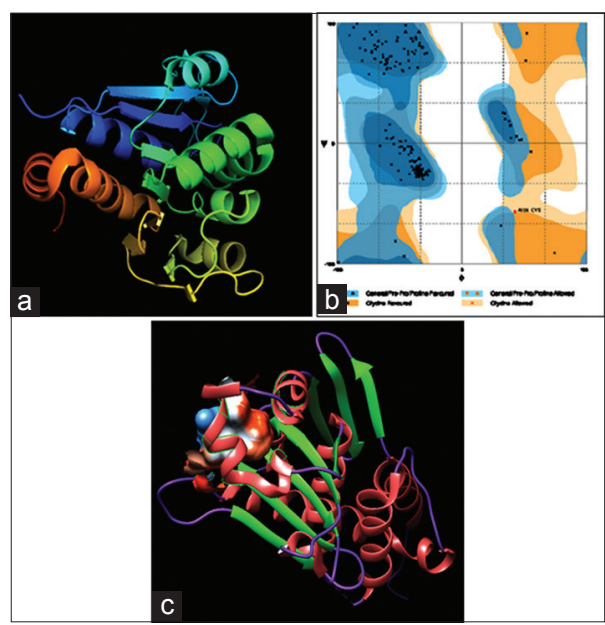

Fig. 3: Three-dimensional (3D) structure, Ramachandran plot, and active site of DJ-1 models. (a) Demonstrates 3D structure of DJ-1, (b) Ramachandran plot was obtained for quality assessment, and (c) Ligand binding site in DJ-1

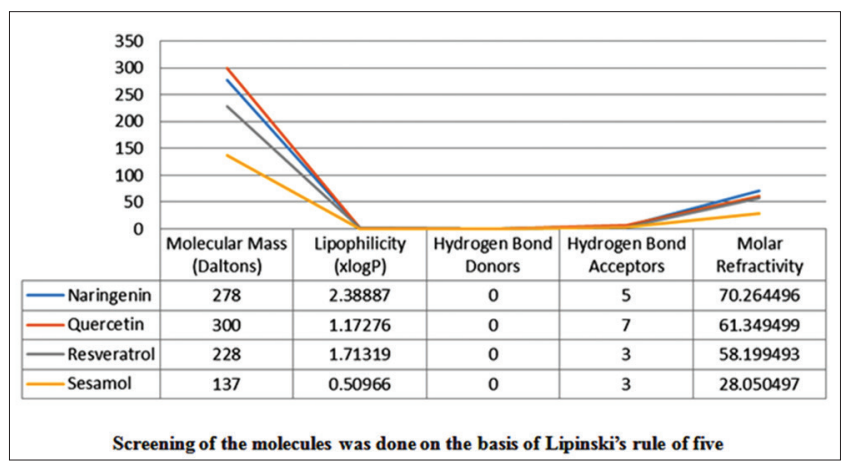

Fig. 4: Druglikeness prediction using Lipinski's filter analysis 
Table 4: Physicochemical properties of ligands

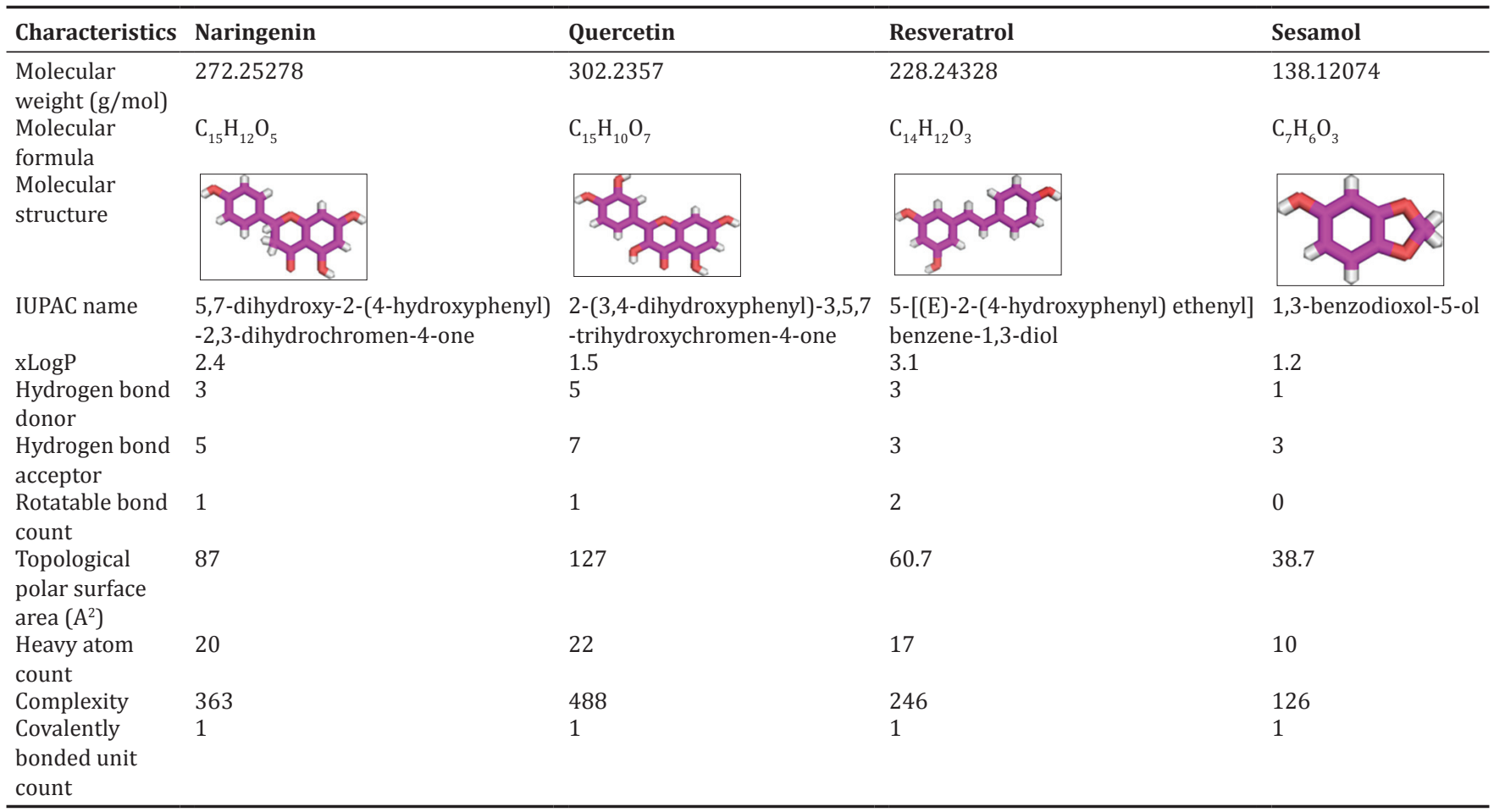

Table 5: Docking calculation of compounds with DJ-1

\begin{tabular}{|c|c|c|c|c|c|c|c|}
\hline $\begin{array}{l}\text { Compound } \\
\text { name }\end{array}$ & $\begin{array}{l}\text { Estimated } \\
\text { free energy of } \\
\text { binding (kcal/mol) }\end{array}$ & $\begin{array}{l}\text { Estimated } \\
\text { binding } \\
\text { constant }\end{array}$ & $\begin{array}{l}\text { Estimated } \\
\text { intermolecular } \\
\text { energy (kcal/mol) }\end{array}$ & $\begin{array}{l}\text { vdW+Hbond+desolv } \\
\text { energy (kcal/mol) }\end{array}$ & $\begin{array}{l}\text { Electrostatic } \\
\text { energy } \\
(\mathrm{kcal} / \mathrm{mol})\end{array}$ & $\begin{array}{l}\text { Estimated } \\
\text { internal } \\
\text { energy } \\
\text { (kcal/mol) }\end{array}$ & $\begin{array}{l}\text { Torsional } \\
\text { free energy } \\
\text { (kcal/mol) }\end{array}$ \\
\hline Naringenin & -4.19 & $851.70 \mu \mathrm{M}$ & -5.38 & -5.25 & -0.13 & +9.69 & +1.19 \\
\hline Quercetin & -3.97 & $1.24 \mathrm{mM}$ & -5.76 & -5.54 & -0.22 & +9.44 & +1.79 \\
\hline Resveratrol & -3.24 & $4.25 \mathrm{mM}$ & -5.03 & -4.91 & -0.12 & +17.09 & +1.79 \\
\hline Sesamol & -3.08 & $5.51 \mathrm{mM}$ & -3.38 & -3.12 & -0.26 & +0.32 & +0.30 \\
\hline
\end{tabular}
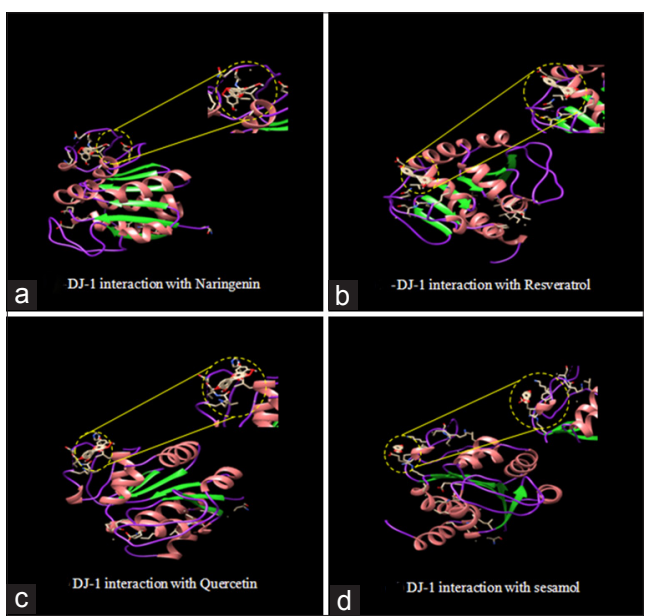

Fig. 5: Docking study of DJ-1 protein with selected compounds. (a) DJ-1 interaction with naringenin, (b) DJ-1 interaction with resveratrol, (c) DJ-1 interaction with quercetin, and (d) DJ-1 interaction with sesamol

their identified catalytic sites have been shown in (Table 6) and their two-dimensional and 3D pattern of interaction is presented in (Fig. 6).

\section{DISCUSSION}

Despite the knowledge of various factors which contribute in the occurrence and progression of PD, the exact cause and cure remains elusive. Mutations in PD-associated genes potentially lead to autosomal dominant ( $\alpha$-synuclein and LRRK2), or autosomal recessive PD (Parkin, PINK1, DJ1, and ATP13A2), respectively [17]. Moreover, these genes display characteristic ubiquitin E3 ligase activity. DJ-1 is ubiquitously expressed in a number of pathways associated with PD pathogenesis and has ubiquitin E3 ligase activity which also reduces $\alpha$-synuclein aggregation [18]. Thus, it seems imperative to design therapeutic strategies aimed at elevating the level of DJ-1 to improve neuronal survival in PD. Further, various studies have advocated that several compounds of plant origin possess neuroprotective properties, however, their mode of action have not been clearly defined [19]. Based on docking study analysis, the present study provides scientific evidence that given four biomolecules, namely, naringenin, quercetin, resveratrol, and sesamol are interacting at the reported binding site of DJ-1. Further, binding constant, $\mathrm{K}_{\mathrm{b}}$ of naringenin, quercetin, resveratrol, and sesamol for DJ-1 was found to be $851.70 \mu \mathrm{M}, 1.24 \mathrm{mM}$, $4.25 \mathrm{mM}$, and $5.51 \mathrm{mM}$, respectively, suggesting that all the selected compounds might be effective as activators of E3 ligase activity of DJ-1. Furthermore, investigation of binding sites within DJ-1 gives a better idea for a valuable drug target site with highest binding and interaction affinity. Based on in vivo experimentation, the most effective compound in modulating E3 ligase activity of DJ-1 was found to be naringenin 
Table 6: DJ-1 known binding site and selected compounds interacting residues

\begin{tabular}{|c|c|}
\hline Compound & Interacting residues \\
\hline Reported & Pro $^{109}$, Thr $^{110}$, Leu $^{112}$, Leu $^{113}$, Ala $^{114}, \mathrm{Glu}^{116}$, Val $^{123}$, Thr $^{125}$, \\
\hline catalytic & 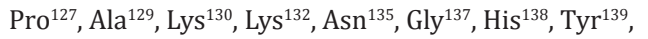 \\
\hline site & $\mathrm{Tyr}^{141}, \mathrm{Glu}^{143}$, and $\mathrm{Arg}^{156}$ \\
\hline Naringenin & $\mathrm{Thr}^{110}, \mathrm{Leu}^{113}, \mathrm{Ala}^{114}, \mathrm{Glu}^{116}, \mathrm{Lys}^{132}$, and Asn ${ }^{135}$ \\
\hline Quercetin & Leu $^{113}$, Ala $^{114}$, Lys $^{132}$, Asn $^{135}$, and His ${ }^{138}$ \\
\hline Resveratrol & $\mathrm{Leu}^{113}, \mathrm{Glu}^{116}$, and $\mathrm{Asn}^{135}$ \\
\hline Sesamol & 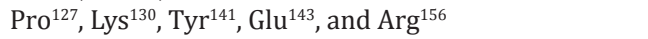 \\
\hline
\end{tabular}
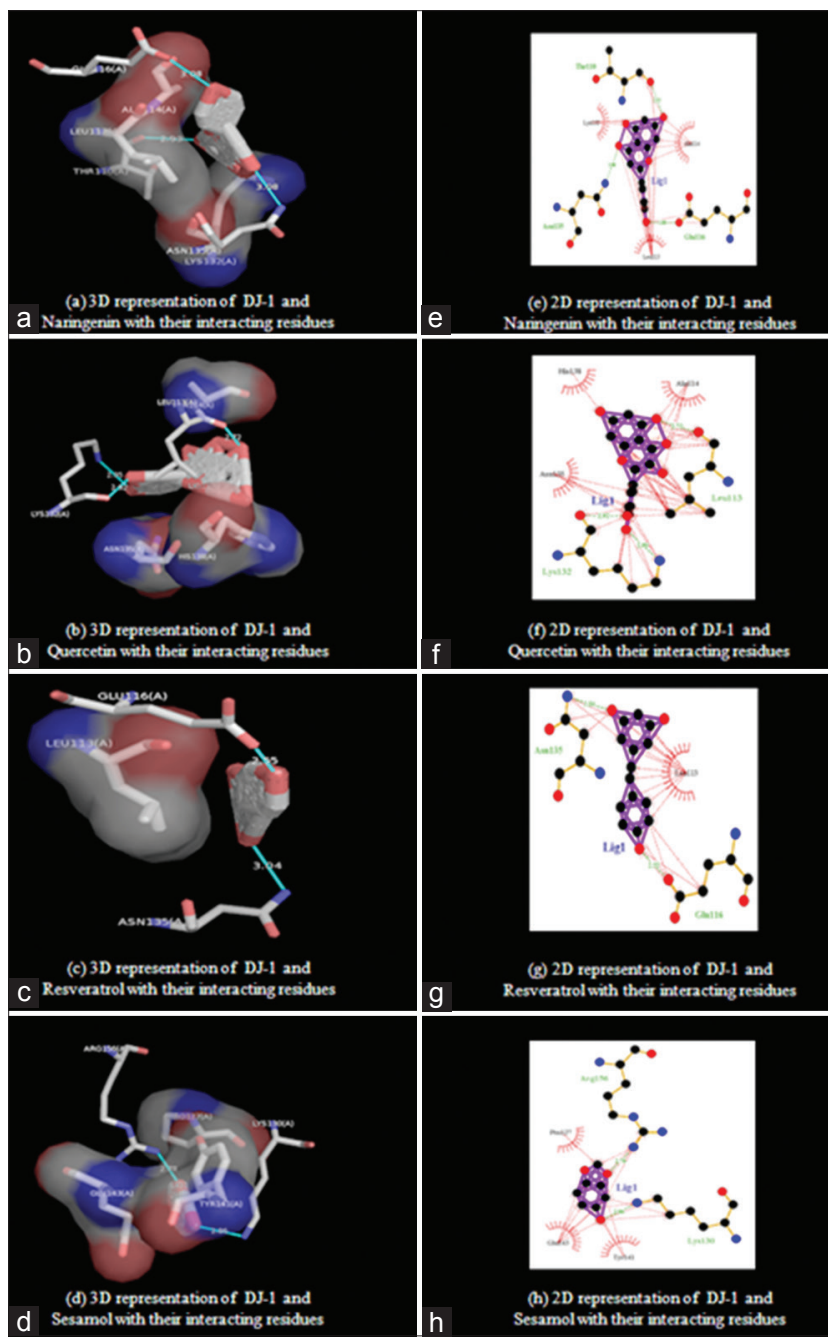

Fig. 6: Binding site of DJ-1 with selected compounds along with its known stimulatory active site. (a) Three-dimensional (3D) presentation of $\mathrm{DJ}-1$ and naringenin with their interacting residues, (b) 3D representation of DJ-1 and quercetin with their interacting residues, (c) 3D representation of DJ-1 and resveratrol with their interacting residues, (d) 3D representation of DJ-1 and sesamol with their interacting residues, (e) twodimensional (2D) representation of DJ-1 and naringenin with their interacting residues, (f) 2D representation of DJ-1 and resveratrol with their interacting residues, (g) 2D representation of DJ-1 and resveratrol with their interacting residues, and

(h) 2D representation of $\mathrm{DJ}-1$ and sesamol with their interacting residues

having minimum binding constant $\mathrm{K}_{\mathrm{b}}$ and highest negative free energy of binding with maximum interacting surface area in a course of docking studies [20-25].

\section{CONCLUSION}

In this study, the sequence and structure analysis of ubiquitin E3 ligase protein DJ-1 were done by various computational tools and softwares. Molecular docking study advocated naringenin to be the most effective compound in elevating E3 ligase action of DJ-1 based on highest negative free energy of binding, minimum inhibition constant Ki, and maximum interacting surface area among the given four biomolecules. Such biomolecules can be effectively used to validate in vitro and in vivo prosurvival outcomes in PD models as well as in clinical scenario. Knowledge gained from this study can be used in broad screening of neuroprotective biomolecules and can be further implemented in designing effective therapeutics for PD.

\section{ACKNOWLEDGMENTS}

The authors would like to thank senior management of Delhi Technological University (DTU) for their encouragement and support. The authors would also like to thank DTU for providing fellowship (DTU-SRF) to SKJ.

\section{REFERENCES}

1. Massano J, Bhatia KP. Clinical approach to Parkinson's disease: Features, diagnosis, and principles of management. Cold Spring Harb Perspect Med 2012;2(6):a008870.

2. Gundersen V. Protein aggregation in Parkinson's disease. Acta Neurol Scand Suppl 2010;190:82-7.

3. Exner N, Lutz AK, Haass C, Winklhofer KF. Mitochondrial dysfunction in Parkinson's disease: Molecular mechanisms and pathophysiological consequences. EMBO J 2012;31:3038-62.

4. Pirkevi C, Lesage S, Brice A, Basak AN. From genes to proteins in mendelian Parkinson's disease: An overview. Anat Rec (Hoboken) 2009;292(12):1893-901.

5. Cookson MR. Parkinsonism due to mutations in PINK1, parkin, and DJ-1 and oxidative stress and mitochondrial pathways. Cold Spring Harb Perspect Med 2012;2(9):a009415.

6. Saurabh S, West AB. The therapeutic potential of LRRK2 and A-synuclein in Parkinson's disease. Antioxid Redox Signal 2009;11(9):2167-87.

7. Klein C, Westenberger A. Genetics of Parkinson's disease. Cold Spring Harb Perspect Med 2012;2(1):a008888.

8. Saikat S, Chakraborty R, De B, Mazumder J. Free radicals, antioxidants, diseases and phytomedicines: Current status and future prospect. Indian J Pharm Sci 2010;3(1):91-100.

9. Jeremy PE. The interactions of flavonoids within neuronal signalling pathways. Genes Nutr 2007;2(3):257-73.

10. Jha SK, Jha NK, Kar R, Ambasta RK, Kumar P. p38 MAPK and PI3K/ AKT signalling cascades in Parkinson's disease. Int J Mol Cell Med 2015;4(2):67-86

11. Lobo V, Patil A, Phatak A, Chandra N. Free radicals, antioxidants and functional foods: Impact on human health. Pharmacogn Rev 2010;4(8):118-26

12. Altschul SF, Gish W, Miller W, Myers EW, Lipman DJ. Basic local alignment search tool 1990;215(3):403-10.

13. Edgar RC. Muscle: A multiple sequence alignment method with reduced time and space complexity. BMC Bioinformatics 2004;5:113.

14. Arnold K, Bordoli L, Kopp J, Schwede T. The SWISS-MODEL workspace: A web-based environment for protein structure homology modelling. Bioinformatics 2006;15;22:195-201

15. Lipinski CA. Lead - And drug-like compounds: The rule-of-five revolution. Drug Discov Today Technol 2004;1(4):337-41.

16. Dundas J, Ouyang Z, Tseng J, Binkowski A, Turpaz Y, Liang J. CASTp: Computed atlas of surface topography of proteins with structural and topographical mapping of functionally annotated residues. Nucleic Acids Res 2006;34:116-8.

17. Wang C, Ma H, Feng X, Xie S, Chan P. Parkin dosage mutations in patients with early-onset sporadic and familial Parkinson's disease in Chinese: An independent pathogenic role. Brain Res 2010;1358:30-8.

18. Mizuno Y, Fisher A, Hanin I. Progress in familial Parkinson's disease. Parkinson's Disease and Related Disorders. Vienna: Springer; 2006; p. 191-204.

19. Kumar GP, Khanum F. Neuroprotective potential of phytochemicals. Pharmacogn Rev 2012;6(12):81-90.

20. Azam F, Madi AM, Ali HI. Molecular docking and prediction of 
pharmacokinetic properties of dual mechanism drugs that block MAO-B and adenosine A(2A) receptors for the treatment of Parkinson's disease. J Young Pharm 2012;4(3):184-92.

21. Gupta P, Rai N, Gautam P. Anticancer drugs as potential inhibitors of acrab-tolc of multidrug resistant Escherichia coli: An in silico molecular modeling and docking study. Asian J Pharm Clin Res 2015;8(1):351-8.

22. Dharani RS, Ranjitha R, Sripathi R, Alimuhammad KS, Ravi S. Docking studies in target proteins involved in antibacterial actionmechanisms: Alkaloids isolated from Scutellaria genus. Asian J Pharm Clin Res 2016;9(5):121-5.
23. Engels M, Balaji BS, Divakar S, Geetha G. Ligand based pharmacophore modeling, virtual screening and molecular docking studies to design novel pancreatic lipase inhibitors. Int J Pharm Pharm Sci 2017;9(4):48-64.

24. Guerrero RV, Vargas RA, Petricevich VL. Chemical compounds and biological activity of an extract from Bougainvillea $x$ Buttiana (var. Rose) holttum and standl. Int J Pharm Pharm Sci 2017;9(4):42-6.

25. Singh N, Kumar B, Kolli MK, Kumari M, Mishra AK. Molecular docking studies of some novel thiophene carbohydrazide derivatives on enterotoxin of bacillus cereus. Int J Curr Pharm Res 2016;8(3):59-63. 